\title{
Several considerations about a pulsed flying spot method implemented with IR thermography
}

\author{
by L. Gavérina*, JC Batsale*, C. Pradere* and D. Mourand**
}

* I2M-TREFLE, UMR CNRS 5295, Esplanade des arts et métiers 33405 Talence Cedex, France, ludovic.gaverina@u-bordeaux.fr

** Epsilon Fahrenheit, ENSAM, Esplanade des Arts et Métiers, F-33405 Talence Cedex, France

\begin{abstract}
A mathematical method to process data provided by a flying spot technique is studied here, by considering the temperature response field around a unique spot. The progress of optical control, laser and IR Camera allow to measure $2 D$ temperature fields localised on a small area compared to the whole recorded image. The study is focused on the logarithmic expression of the in-plane temperature field in order to analyse and to measure different parameters such as the in plane thermal diffusivity and the localization of the spot. As a perspective, this method can also be used to detect cracks parallel or perpendicular to the surface.
\end{abstract}

\section{Introduction}

The use of this new scanning systems (based on galvo-mirror) allows to easily control the movement of a laser hot spot over a plane surface. It is then suitable to develop "new flying spot methods" as alternative methods compared to the initial "Flying spot" technique [1] based on a constant velocity of the spot.

On the other hand, instead of a single temperature measurement used in [1], IR thermography allows to process temperature fields in order to process the complete transient temperature field in a small area around the spot. Even if a great number of pulsed spots should be deposited, the preliminary step will consist in analyzing the effect of a unique spot. In order to implement such a processing, it is proposed to analyze the transient field of the temperature response from the analytical expression. Such a method is very near and complementary of the TSR method used in the case of 1D flash experiment [2], because the analysis is done by considering the logarithm of the temperature versus the logarithm of time.

In this paper, firstly, some theoretical aspects are considered. Then, a new estimation method will be presented and finally the first results of experimental data are shown to validate the capability of the method by esstimating the in plane diffusivity, the position of the spot and the nature of the transverse heat transfer.

\section{Theoretical considerations on a simple analytical case}

The analytical expression of the temperature response field of a pulsed punctual heat source, imposed on the front face $(z=0)$ of a homogeneous semi-infinite media is written as follows:

$T(x, y, z=0, t)=\frac{Q}{\rho c} \frac{\exp \left(-\frac{\left(x-x_{0}\right)^{2}}{4 a_{x} t}\right)}{\sqrt{\pi a_{x} t}} \frac{\exp \left(-\frac{\left(y-y_{0}\right)^{2}}{4 a_{y} t}\right)}{\sqrt{\pi a_{y} t}} \frac{1}{\sqrt{\pi a_{z} t}}$

Where:

$$
\begin{aligned}
& a_{x}, a_{y}, a_{z}: \text { Thermal diffusivities versus } x, y, z \text { direction }\left(m^{2} \cdot s^{-1}\right) \\
& Q: \text { Energy of the pulse point source }(\mathrm{J}) \\
& \rho c: \text { Volumic heat capacity }\left(J \cdot m^{-3} \cdot K^{-1}\right)
\end{aligned}
$$

One other expression can be considered in the case of thin samples where the temperature distribution versus

the z-direction can be assumed as uniform. It yields a theoretical 2D expression:

$$
T(x, y, t)=\frac{Q}{\rho c} \frac{\exp \left(-\frac{\left(x-x_{0}\right)^{2}}{4 a_{x} t}\right)}{\sqrt{\pi a_{x} t}} \frac{\exp \left(-\frac{\left(y-y_{0}\right)^{2}}{4 a_{y} t}\right)}{\sqrt{\pi a_{y} t}}
$$


Such an expression can be expressed as a product of 3 responses related to each other in 1D diffusion.

If the space average following the $y$ direction is considered it yields a $1 \mathrm{D}$ temperature distribution such as:

$\langle T\rangle_{y}(x, t)=\frac{1}{L} \int_{O}^{L} T(x, y, z=0, t) d y$

With $0<<y_{0}<<L$

The consideration of the Logarithm of the previous expression then yields:

$\ln \left(\langle T\rangle_{y}(x, t)\right)=\ln \left(\frac{k}{t^{\alpha}}\right)-\frac{\left(x-x_{0}\right)^{2}}{4 a_{x} t}$ Where $\left\{\begin{array}{l}\alpha=\frac{1}{2} \text { for thin layer versus z direction } \\ \alpha=1 \text { for semi - infinte medium versus z direction }\end{array}\right.$

With $k$ : Arbitrary constant

The expression can be assimilated to a polynomial expression such as:

$\ln \left(\langle T\rangle_{y}(x, t)\right)=a_{0}(t)+a_{1}(t) x+a_{2}(t) x^{2}$

With special behavior of $a_{0}(t), a_{1}(t), a_{2}(t)$

$$
\left\{\begin{array}{l}
a_{2}(t)=-\frac{1}{4 a_{x} t} \\
a_{1}(t)=\frac{x_{0}}{2 a_{x} t} \\
a_{0}(t)=-\frac{x_{0}{ }^{2}}{4 a_{x} t}+\ln \left(\frac{k}{t^{\alpha}}\right)
\end{array}\right.
$$

Several comments flows from the previous expressions:

- The $a_{2}(t)$ is only dependent on the thermal diffusivity $(a)$. It is then a suitable way to verify the in-plane homogenous transfer at different time steps independent of the transverse heat transfer and the position of the spot.

- $\quad$ Once, the in-plane diffusivity is estimated, it is suitable to estimate the position of the center of the spot $x_{0}$. with the consideration of $a_{1}(t)$.

- The estimation of $a_{0}(t)$ is directly related to a 1D front face flash experiment such signal contribute to process for example the TSR method [2] used here to estimate the parameter on a $\ln (T)$ versus $\ln (t)$ graph.

\section{Different steps for the temperature fields processing and parameter estimation}

\subsection{Preliminary steps for the temperature fields processing}

From the previous theoretical considerations it is necessary to validate the different steps of the parameters estimation (4).

- Offset estimation:

The initial offset level must be accurately estimated before every experiment. The space average of the completely uniform but noisy temperature field must be implemented before the heat pulse. Such an estimation is important because the logarithmic scale is influenced by zeros or negative values of the data. 
- Space separation

In order to verify that the temperature distribution $(1 . b)$ is separable such as:

$T(x, y, z=0, t)=X(x, t) * Y(y, t)$.

It is suitable to consider Singular Value Decomposition (SVD) of instantaneous temperature field at each time step $[3,4$, and 5].

$$
T(x, y, t)=\sum_{k=1}^{N} \gamma_{k}\left(U_{k}(x, t) V_{k}(y, t)\right)
$$

$U_{k}(x, t)$ and $V_{k}(y, t)$ are orthogonal functions and $\gamma_{k}$ are the singulars values in decreasing mode. If the first singular value is much greater than the others, the temperature field can be assumed to be separable such as:

$T(x, y, t)=\gamma_{1} U_{1}(x, t) V_{1}(y, t)$

The $U_{1}(x, t)$ can be processed with the help of the theoretical consideration of the section (4).

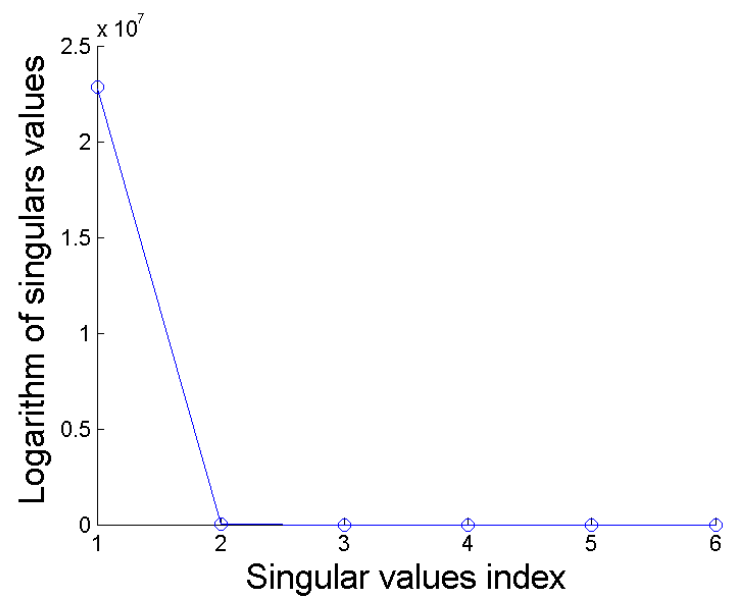

Fig. 1: Diagonal of singular values related to the expression (6) 
- $\quad$ Thresholding

It is important to process only the signal where the time and space derivative of the temperature field is non-zero. Several methods such as the consideration of the correlation between the time derivative and the laplacian of the temperature field (see [4]), can be considered. A simple threshold greater than the measurement noise can also be considered.

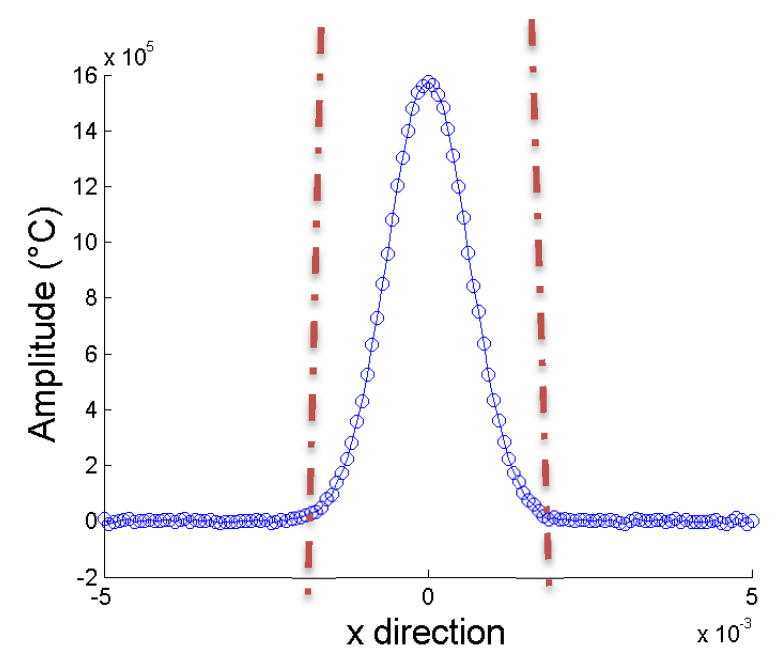

Fig. 2: Temperature profile (blue line) and thresholding (red dotted line)

\subsection{The parameters estimation}

For convenient reasons, the measuremnt noise will be considered as uniform on the $\ln (T)$ distribution such as:

$\ln \left(\hat{T}(x, t)_{i}\right)=\ln \left(T\left(x, t_{i}\right)\right)+e_{T\left(x, t_{i}\right)}$

with $e_{T\left(t_{i}\right)}$ : random fluctuation which is added to a signal $\ln \left(T\left(x, t_{i}\right)\right)$ and considered with zero mean and uniform standard deviation (diagonal covariance matrix). From the measurements, a polynomial fitting can be implemented at each time $t$.

The parameter ${ }^{\hat{\beta}_{n}}$ can be estimated by using a linear least-square relation. It can be expressed as a linear combination of temperature field $\ln (\hat{T}(x, t))$ with parameter $\hat{\beta}$ is $\ln (\hat{T}(x, t))=X \hat{\beta}$.

$\left[\begin{array}{c}\ln \left(\hat{T}_{1}\right) \\ \vdots \\ \ln \left(\hat{T}_{N}\right)\end{array}\right]_{t}=\left[\begin{array}{ccc}1 & x_{1} & x_{1}^{2} \\ \vdots & \vdots & \vdots \\ \vdots & x_{N} & x_{N}^{2}\end{array}\right]\left[\begin{array}{c}\hat{\beta}_{1} \\ \vdots \\ \hat{\beta}_{n}\end{array}\right] \Leftrightarrow\left[\begin{array}{c}\ln \left(\hat{T}_{1}\right) \\ \vdots \\ \ln \left(\hat{T}_{N}\right)\end{array}\right]_{t}=\left[\begin{array}{ccc}1 & x_{1} & x_{1}^{2} \\ \vdots & \vdots & \vdots \\ \vdots & x_{N} & x_{N}^{2}\end{array}\right]\left[\begin{array}{c}\hat{a}_{0}(t) \\ \hat{a}_{1}(t) \\ \hat{a}_{2}(t)\end{array}\right]$

The optimal estimation yields then: 


$$
\Leftrightarrow\left[\begin{array}{c}
\hat{a}_{0}(t) \\
\hat{a}_{1}(t) \\
\hat{a}_{2}(t)
\end{array}\right]=\left(X^{t} X\right)^{-1} X^{t}[\ln (\hat{T})]
$$

\section{Test of the estimation method on numerical experiments}

A numerical simulation in the case of an homogeneous isotropic sample of thermal diffusivity $a=10^{-6} \mathrm{~m}^{2} . \mathrm{s}^{-1}$, heated in the center $x_{0}=5 \mathrm{~mm}$ by one heat pulse at $t_{0}=0$. is considered. The thickness of the sample is $e=10^{-2} \mathrm{~m}^{2} \cdot \mathrm{s}^{-1}$. Instead of in expression (8), a measurement noise with a uniform standard deviation is added to the temperature signal. The amplitude of the noise is at about $1 \%$ of the initial maximum of the temperature field.

In this case, one polynomial fit $\ln (\hat{T}(x, t))=\sum_{n=0}^{2} x^{n} \hat{\beta}_{n}$ is used to determine the different polynomial coefficients. It was applied to different temperature fields in this time.

The parameters $\hat{\beta}_{n}$ can then be processed at every time step. On the fig.3a is plotted the estimation result of $1 / a_{2}(t)$. It can be verified that this estimation is following the linear dependence on time (the noise effect dispersion is expected for long times). On the fig. $3 \mathrm{~b}$ is plotted the estimation result of $1 / a_{1}(t)$, as previously is linearly depended on time and the noise increase for long time.

(a)

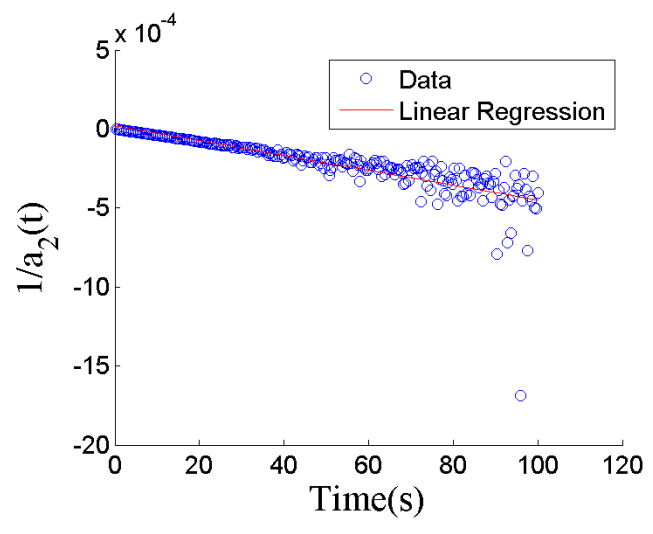

(b)

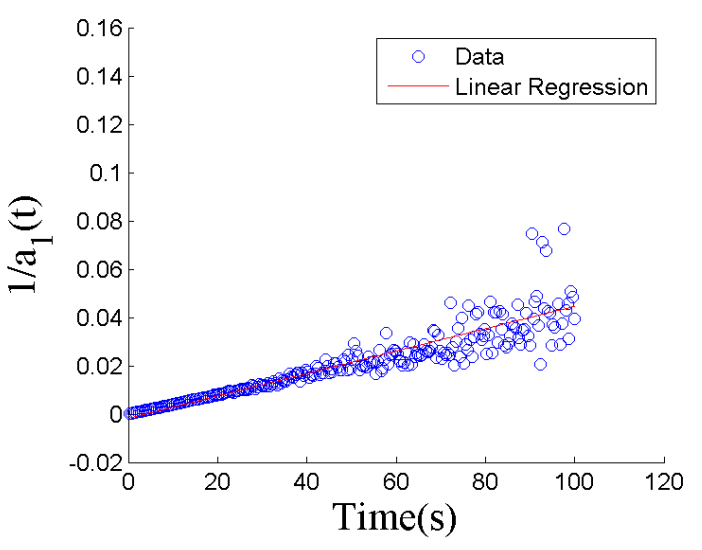

Fig. 3: a: Coefficient of the quadratic term (blue circle) and linear regression (red line). From the slope of this graph, the thermal diffusivity can be estimated. In this case, it was estimated a=1.048e- $6 \mathrm{~m}^{2} / \mathrm{s}$ (error:4.5802\%) $b$ : Coefficient of the linear term (blue circle) and linear regression (red line). From the slope of this graph, the position of the laser spot can be estimated. In this case, this position is estimated $\times 0=0.0050 \mathrm{~m}$ (error lower than $1 \%$ ).

The estimation of the paraemter $a_{0}(t)$ can be considered in the complete $2 \mathrm{D}$ or $3 \mathrm{D}$ case by the reconstruction of the $2 \mathrm{D}$ signal and by plotting the maximum temperature at the estimated coordinates ( $\left.x_{0}, y_{0}\right)$. The figure 4 is showing that the reconstructed signal is observing fairly the -1 slope in a case of $2 \mathrm{D}$ heat diffusion (case of a thin sample). This last estimation step allows to completely validate the expressions (4) and to analyse the nature of the transverse heat transfer. As a perspective, a lot of other situations of transverse transfer can be approached (3D case with delaminations, multilayered cases etc...). 


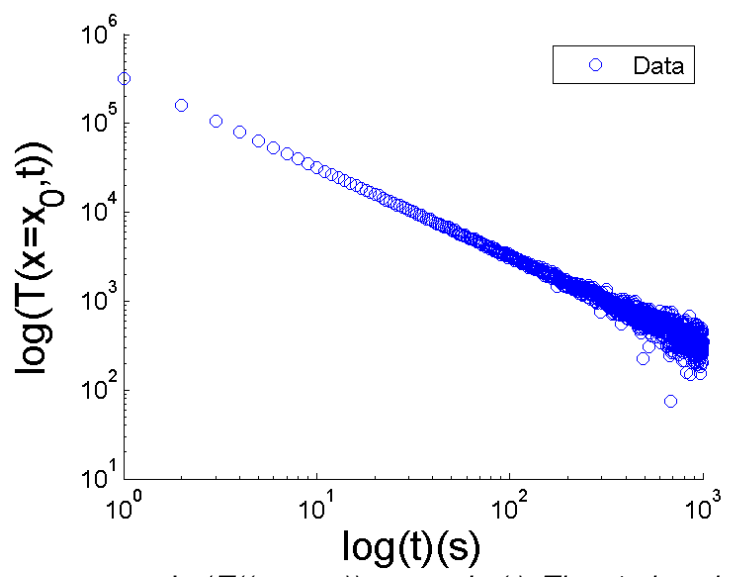

Fig.4: Logarithm of the maximum temperature $\operatorname{Ln}\left(T\left(\left(x_{0}, y_{0}, t\right)\right)\right.$ versus $\operatorname{Ln}(t)$. The -1 slope is well observed at short time.

\section{Test of the estimation method on numerical experiments}

\subsection{Set-up}

The laser excitation is provided by a fiber laser. This type of laser has a wavelength of $976 \mathrm{~nm}$, and is equipped with a collimation system (Thorlabs). The deviation device is used to change the direction of the laser beam, for that it uses one Dual-Axis Scanning Galvo Systems (Thorlabs GVS112 (/M)). The principle of the laser beam deviation towards the sample is shown in Fig.5.

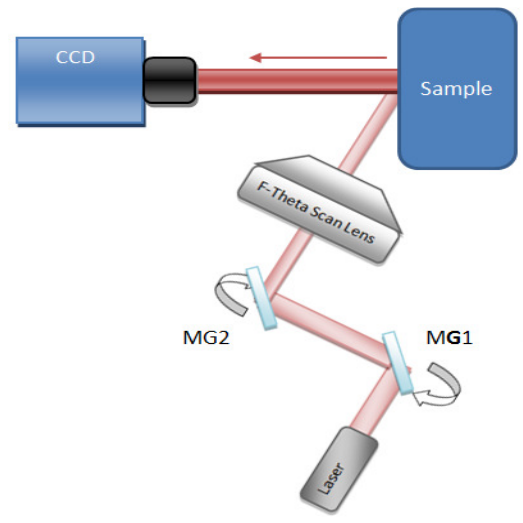

Fig. 5:Set-up flying spot 
The laser beam passes through the lens 1 and arrives to a mirror situated in the deviation device. Due to the mirror, the beam changes its direction and continues its path until the lens.2. By passing through the lens 2 , the beam gets out of the device and goes towards the sample. The lens 2 enlarges the beam diameter as a function of the distance $\mathrm{d}$, which allows heating the samples in a large surface area as expected. For measuring the temperature fields, IR camera MCT (FLIR SC7000) is used.

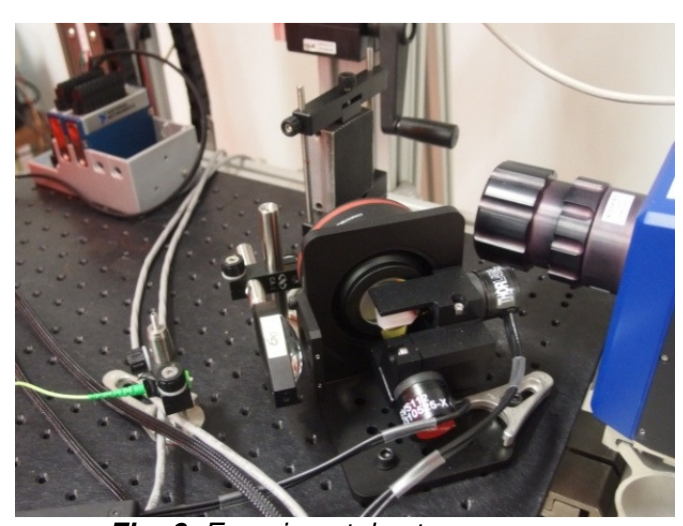

Fig. 6: Experimental set-up

\subsection{Experimental results and discussion}

An experimental validation is performed with the previous configuration. The spatial resolution is about $200 \mu \mathrm{m}$ with $\Delta x=\Delta y$ for each pixel and the acquisition frequency is $50 \mathrm{~Hz}(\Delta t=20 \mathrm{~ms})$. The sample Plexiglas ${ }^{\circledR}$ with the thickness of $3 \mathrm{~mm}$ and $\mathrm{L}=60 \mathrm{~mm}$ and $\mathrm{I}=40 \mathrm{~mm}$. The transverse thermal diffusivity is $1.25^{*} 10^{-7} \mathrm{~m}^{2} . \mathrm{s}^{-1}$. The sample surface is covered with a thin black paint in order to have the same absorption all over the surface.

For the separation of the $x$ and $y$ directions of tranfer, the marginal space averaging is considered such as:

$$
\left\{\begin{array} { l } 
{ \langle T \rangle _ { y } ( x , t ) = \frac { 1 } { L } \int _ { O } ^ { L } T ( x , y , z = 0 , t ) d y } \\
{ \langle T \rangle _ { x } ( y , t ) = \frac { 1 } { L } \int _ { O } ^ { L } T ( x , y , z = 0 , t ) d x }
\end{array} \Leftrightarrow \left\{\begin{array}{l}
\langle T\rangle_{y}(x, t)=\frac{k}{t^{\alpha}} e^{\left(-\frac{\left(x-x_{0}\right)^{2}}{4 a_{x} t}\right)} \\
\langle T\rangle_{x}(y, t)=\frac{k}{t_{\alpha}} e^{\left(-\frac{\left(x-x_{0}\right)^{2}}{4 a_{x} t}\right)}
\end{array}\right.\right.
$$




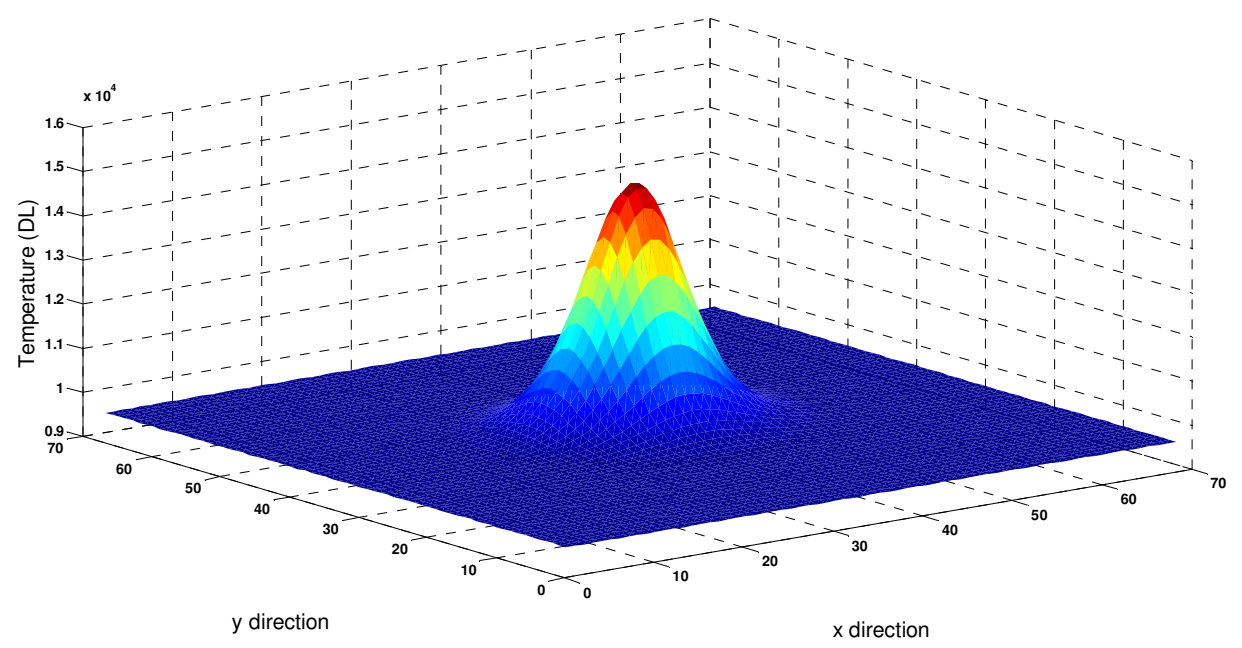

Fig. 7: Measured temperature field just after heat pulse semi-infinite behaviour.

From this IR measurement, the estimations of the parameters $\hat{\beta}_{n}$ along both axes are plotted in fig. 8 , fig. 9 and fig 10, similarly as in the case of the numerical simulation. The figure 8 and 9 related to the estimation of the thermal diffusivity and the position of the spot are perfectly reproducing the theoretical estimation process (good accuracy at short times for the diffusivity and the spot position estimation). Additionally, the 2 directions of the transfer following $x$ and $y$ can be compared and give accurate results.

The figure 10 is representing the evolution of the logarithm of the maximum temperature. The -1 slope is quite well observed at intermediary times corresponding to a purely in-plane heat transfer. The very short times seems to verify a $-3 / 2$ slope. This result will be more accurately studied in the future. The long times are noisy and biased. This bias is likely due to convective losses not taken into account in the present model and is also strongly depending on the offset estimation.

Such estimations steps are necessary in order to extend the method to defect detection even in each $x, y$ or $z$ direction. A crack or a defect will appear at a characteristic time and perturbate the curves here presented. It will be a good way to detect anomalies. 


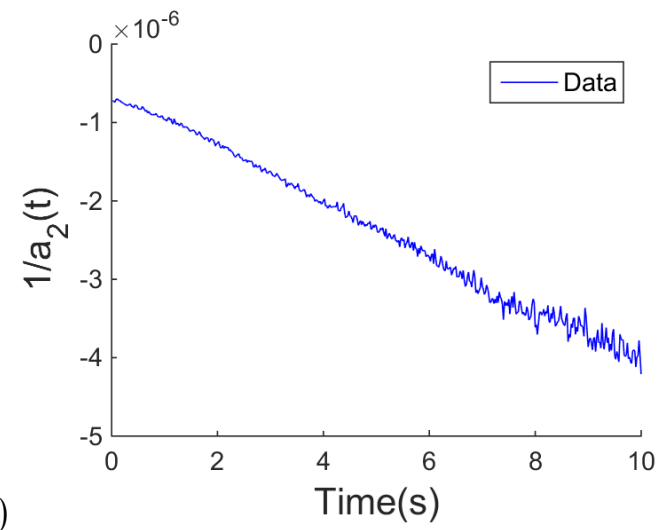

(a)

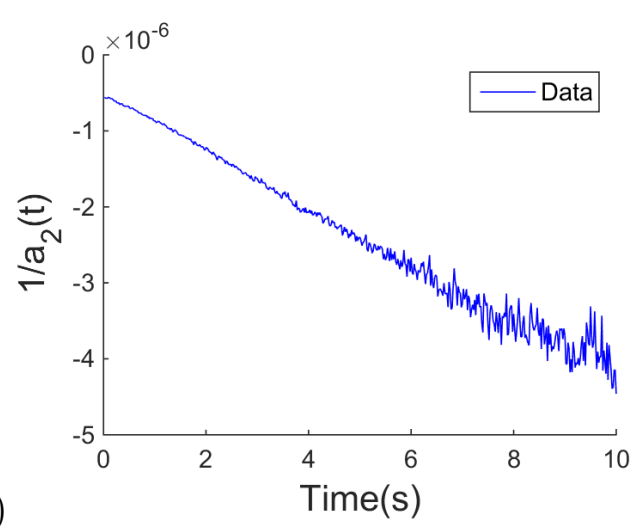

Fig. 8: $a$ and $b$ : Coefficient of the quadratic term (blue line) along $x$ direction and along $y$ direction . In this case, it was estimated $a x=1.3254 \mathrm{e}-07 \mathrm{~m}^{2} / \mathrm{s}$ (error: $5.7 \%$ ) and $a y=1.3198 \mathrm{e}-07 \mathrm{~m}^{2} / \mathrm{s}$ (error: $5.3 \%$ ). It can be noticed that for a long time the noise increases and it increases the error on the estimation of the thermal diffusivity .

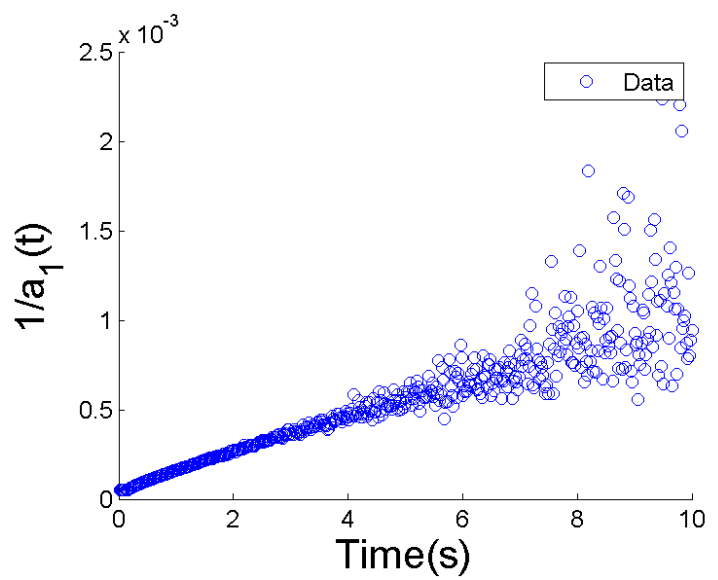

(a)

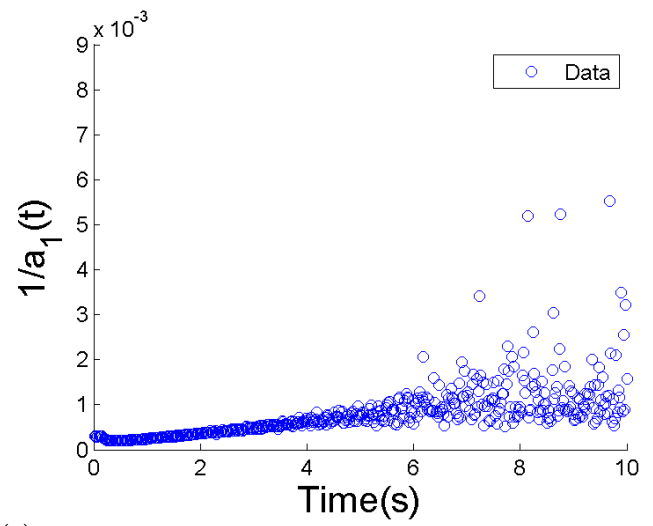

(b)

Fig. 9: $a$ and b: Coefficient of the linear term (blue line) along $x$ direction and along $y$ direction. From the slope of this graph, the position of the laser spot can be estimated. In this case, this position is estimated at $x 0=0.0021 \mathrm{~m}$ (error: $1.9 \%$ ) and $y 0=0.0017 \mathrm{~m}$ (error: $5.9 \%)$ 


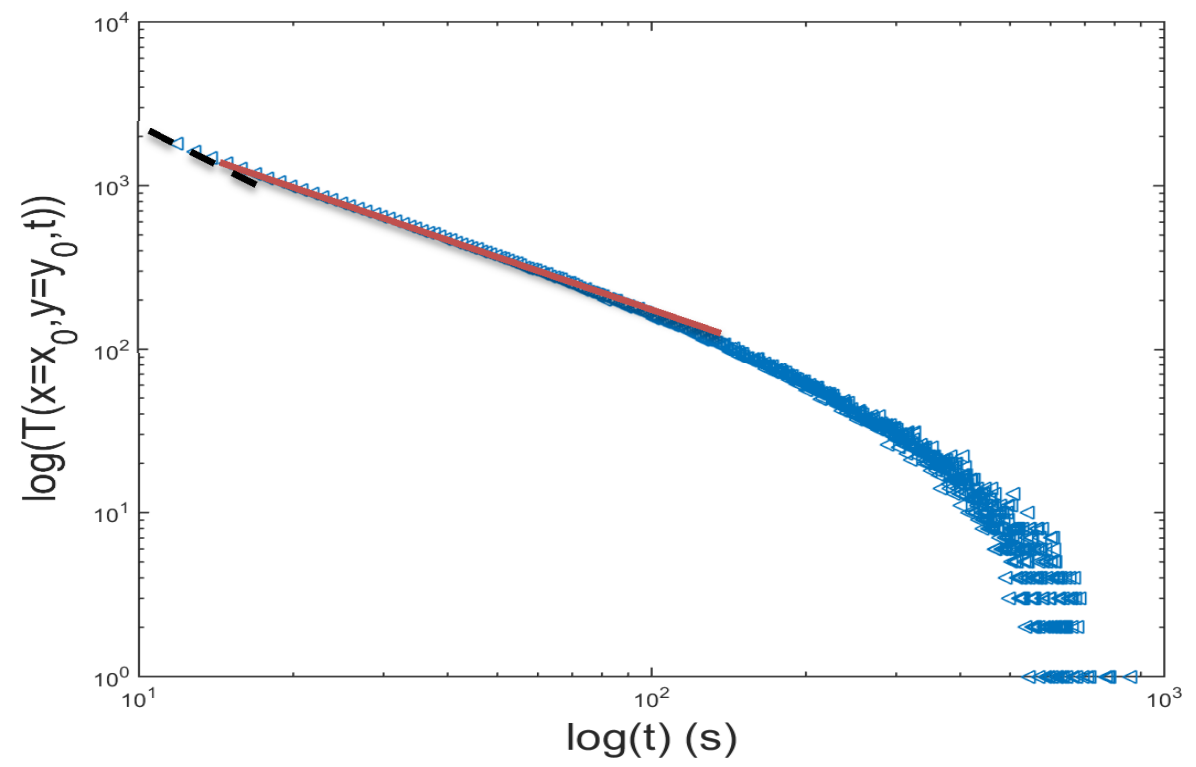

Fig. 10: The logarithm of maximum of the temperature fields (blue circle) and along y direction (green triangle) are plotted versus the logarithm of time. The $-3 / 2$ is well observed at the beginning (black dotted line) and - 1 is well observed after the-1 slope (red line).

\section{Conclusion}

The results presented in this paper are constituting a preliminary step in order to validate a new flying spot method. The data processing method can be considered as an extension of the Shepard method because the estimation is processed by considering logarithmic scales in temperature and in time.

The in-plane diffusivity and the position of the spot is estimated independently on the transverse heat transfer by considering polynomial expressions.

The study and the validation with an homogeneous sample was necessary. In the future, the polynomials can be considered at higher orders for the detection of dissymetries or anomalies related to the Gaussian curves. The perspectives of this work are to test the method in several situations such as: cracks perpendicular or parallel to the observation surface, interfaces, mapping of thermophysical properties, research of a "small" anomaly over a large area...).

\section{REFERENCES}

[1] Krapez J.C., Spatial resolution of the flying spot camera with respect to cracks and optical variations. Proceedings of the 10th international conference on photoacoustic and photothermal phenomena. AIP Conference Proceedings, Volume 463, pp. 377-379, 1999.

[2] Shepard S.M., Advances in pulsed thermography. Thermosense XXIII, Proc. SPIE.; Volume 4630: p 511-515, 2001.

[3] Avayzyan V., Etude de champs de temperature séparables avec une double decomposition en valeurs singulières Quelques applications à la caracterisation de proprieties thermophysiques de matériaux et au contrôle Non-Destructif, Doctorate Thesis, Université de Bordeaux, France 2012

[4] Bamford M., Batsale J.C.: Analytical singular value decomposition of infrared image sequences : microcrack detection on ceramic composites under mechanical stresses. Comptes Rendus Ac. Sci. - Mecanique, 336(5):440-447, 2008.

[5] Batsale JC., Pradere C., Infrared image processing devoted to thermal non-contact characterization- A connecting thread for the I2M-Bordeaux activities in the field of IR thermography, first QIRT Asia Conference, July 2015.

[6] Pradere C., Toutain J., Batsale J.C., Morikawa J., Hayakawa E., Hashimoto T., Microscale thermography of freezing biological cells in view of cryopreservation, QIRT congress, Microscale applications, p 403-411, Krakow, Poland, 2008.

[7] Philippi I., Batsale J.C., Maillet D. and Degiovanni A., Measurement of thermal diffusivities through processing of infrared images. Review of Scientific Instruments, Volume 66(1):p182-192, 1995. 